\title{
Performance Evaluation LoRa-GPRS Integrated Electricity usage Monitoring System for Decentralized Mini-Grids
}

\author{
Shaban Omary ${ }^{1}$, Anael Sam ${ }^{2}$ \\ Department of Communications Science and Engineering \\ The Nelson Mandela African Institution of Science and Technology (NM-AIST), Arusha, Tanzania
}

\begin{abstract}
The emerging Internet of Things (IoT) technologies such as Long-Range (LoRa), combined with traditional cellular communications technologies such as General Packet Radio Service (GPRS) offers decentralized mini-grid companies the opportunity to have cost-effective monitoring systems for the mini-grid resources. Nevertheless, most of the existing decentralized mini-grid companies still rely on traditional cellular networks to fully monitor electricity consumption information, which is not a feasible solution especially for the resource-constrained mini-grid systems. This paper presents the performance evaluation of the proposed LoRa-GPRS integrated power consumption monitoring system for decentralized minigrid centers. Each mini-grid center consists of a network of custom designed smart meters equipped with LoRa modules for local data collection, while the GPRS gateway is used to transmit collected data from the local monitoring centre to the cloud server. Performance testing was conducted by using five electrical appliances whose power consumption data form the cloud server was compared to the same data collected by using a reference digital meter. The correlation between the two data sets was used as a key performance metric of the proposed system. The performance results show that the proposed system has a good accuracy hence providing a cost-effective framework for monitoring and managing of power resources in decentralized mini-grid centers.
\end{abstract}

Keywords-Internet of Things; LoRa; GPRS; decentralized mini-grid systems; electricity usage monitoring

\section{INTRODUCTION}

For many decades, conventional centralized grid extension has been a prevalent mode of electrification in several countries [1]. Nevertheless, in many developing countries, particularly Sub-Saharan Africa (SSA), decentralized mini-grid systems have emerged as alternative to electrification efforts since national grid extensions cannot be extended to all community areas due to high budget requirements [2]. This is also influenced by the nature of community settlements in many parts of the developing world, as the majority of people live in areas far from the national grid network. As a result, numerous countries have formulated Small Power Producers (SPPs) frameworks to encourage private companies to invest in decentralized off-grid systems [3].

One of the challenges facing the existing decentralized mini-grid systems is the lack of cost-effective infrastructure to monitor and manage electricity consumption from remote mini-grid centers [4]. While most off-grid companies manage and run several grid centers across the country from different regions, most of these centers are located in remote areas where access to broadband access technologies is still limited [5]. Most grid utilities still rely on traditional cellular networks to completely control and monitor energy utilization in decentralized grid centers. Nonetheless, each mini-grid center usually has a large number of consumer meters, making it not a cost-effective solution to rely entirely on cellular networks for electricity usage monitoring [6]. The mini-grid company might have to negotiate data plan agreements with cellular network providers to accommodate all the enormous data traffic, depending on the number of customers each mini-grid center serves [7]. This strategy is obviously inefficient since mini-grid companies do not have full control of the communication infrastructure across all the mini-grid centers [8].

Recent developments in the Internet of Things (IoT) provide an incentive for the implementation of heterogeneous networks to more effectively manage remote monitoring systems such as smart cities, weather data and home automation compared to the use of traditional cellular networks [9,10]. Low Power Wide Area Networking (LPWAN) is one of the latest IoT technologies providing long-range communication while using low power to transmit data, which is one of the most important criteria for remote monitoring systems. The most popular LPWAN technologies include Long Range (LoRa), Narrow Band IoT (NB-IoT) and Sigfox [11]. Among these technologies, LoRa is the most adopted because it promises ubiquitous connectivity in outdoor IoT applications, while keeping network management simple [12]. Having higher noise immunity and ability to transmit data over long distance while using license-free spectrum makes LoRa a cost-effective solution for private companies to create a wide area private network for embedded systems [13]. LoRa also has an adaptive data rate capability in the Physical Layer (PHY) which offers competitive advantages for private network deployment in urban areas compared to Sigfox and NB-IoT technologies [14]. By integrating with traditional cellular communication technologies such as General Packet Radio Service (GPRS), LoRa implementation offers a viable solution for the deployment of a cost-effective cloud-based monitoring system for remote, decentralized mini-grid centers especially in developing countries [15].

This paper presents a performance evaluation of the LoRaGPRS integrated electricity usage monitoring system for decentralized mini-grid systems. The proposed system will 
help mini-grid companies to effectively and economically monitor and manage utilization of the available mini-grid resources located in different locations across the country.

The main contributions of this paper are:

- This study proposes a cost-effective electricity power consumption monitoring system for decentralized minigrid systems by integrating LoRa and GPRS technologies. While most of the existing grid companies use Power Line Communication (PLC) as the grid monitoring technology, power stability issues makes this solution to be less practical particularly in developing countries. In this study, the combination of LoRa and GPRS technologies is used to monitor power consumption in grid centers. This approach minimizes operating costs since LoRa uses unlicensed spectrum and low power to transmit data over large distance. A single GPRS gateway is only used to send power consumption data to the cloud server.

- While other studies[16]-[20 ] used different metrics to assess LoRa technology efficiency in IoT applications, the majority evaluated multiple dependent variables at a time that was time-consuming and demanded thorough analysis. In this study, a simple but effective approach is used to assess the performance of the proposed system using power consumption data collected on the cloud server over a specific period of time as the performance metric. The data collected was compared to a data set collected locally by means of a standard reference meter. By using this approach the final collected data takes into account all other communication parameters involved in the system infrastructure.

The reset of the paper is organized as follows. Section II reviews some of the related works in LoRa technology and its performance evaluation in various IoT based monitoring applications. Section III describes materials and methods used in the proposed system. Section IV discusses the approach used for assessing the evaluation of the proposed system. Section V discusses the results and the main findings while conclusion for the study is given in Section VI.

\section{RELATED WORKS}

This section presents review of the recent works related to LoRa implementation in IoT applications, its performance evaluation and their implications. The researchers' methodologies and their main findings are explicitly discussed in order to highlight potential contributions that have been used as benchmark for the implementation of the system proposed in this study.

In [16], authors presented the analysis of LoRa technology performance experimental results by considering various performance metrics such as Transmission Power (TP), Spreading Factor (SF), coverage and data throughput. Using the European Industrial Scientific and Medical (ISM) band of $868 \mathrm{MHz}$, the SF of 12 and the default TP of $14 \mathrm{dBm}(25$ $\mathrm{mW}$ ), the study assessed the robustness of LoRa technology with different configurations such as the use of $125 \mathrm{kHz}$ and
$250 \mathrm{kHz}$ Channel Bandwidths (CB) and various coverage distances from $2 \mathrm{~km}$ to $30 \mathrm{~km}$. The study found that LoRa technology has a good performance for applications that do not need heavy data usage, and is therefore an impressive new technology that can be used in smart metering systems like power, gas and water utilities.

Another study proposed a LoRa-3G hybrid communication architecture for deploying a monitoring system to optimize maintenance operations in electricity and gas distribution systems [17]. The authors carried out a performance test of the effect of different terrain variables on the LoRa communication range. This was accomplished by installing a LoRa transmission node at various locations and distances from the gateway. The test was conducted by varying SF from 7 to 12 , using the European ISM $868 \mathrm{MHz}$ band and $125 \mathrm{kHz} \mathrm{CB}$ while using a $2.7 \mathrm{dBi}$ antenna gain for both gateways and nodes. The study found that the Line of Sight (LoS) is one of the most key factors for LoRa network performance. Therefore, when deploying LoRa network, it was proposed that transmission nodes should be located at high and isolated points to ensure LoS and avoid unnecessary blockages.

In-depth analysis of LoRa technology and its functional components has been presented [18]. Field tests and simulations were used to assess the physical and data link layers of LoRa network. The test was carried out via a testbed by positioning LoRa end devices at various outdoor locations, while the Cisco910 industrial router was placed indoor as a gateway. Using the default transmission power of $14 \mathrm{dBm}$, the test results show that high SF provides better coverage but at a cost of lower data rate. Nonetheless, in actual implementation of LoRa network protocols, SF is usually automatically adjusted to maintain the signal transmission quality. Researchers agreed that hardware resource constraints like power and memory requirements restrict the applicability of conventional cellular networks in the implementation of IoT. Therefore, the researchers concluded that the advent of LoRa technology promises communication requirements suitable for IoT applications, particularly smart metering systems, due to the potential to allow low power consumption and adaptive transmission levels relative to transmission ranges.

In another related study [19], authors provided a review of LoRa technology, its main technological interpretations and its comparison to other traditional approaches in IoT wireless technologies operating in the ISM spectrum. A proof-ofconcept performance testing was carried out by installing a private LoRa network with in order to monitor temperature and humidity across the multi-store building. The researchers found that implementation of the LoRa network offers good performance in IoT applications compared with other wired and wireless technologies. The researchers also found that while LPWAN inherited the basic features of the legacy cellular networks such as user mobility and resource management, the use of a lighter control plane makes LPWAN a preferred candidate technology for low data-rate services such as in smart grid applications.

In [20], proposed a hybrid LoRa and Power Line Communication (LoRa-PLC) IoT sensor network for managing and improving energy metering at the campus using LoRa as 
an outdoor solution and Power Line Communication (PLC) as an indoor solution. In this study, several SX1272 LoRa modules were used as end-devices distributed around office rooms and inside electricity meters. The S7G2 starter kit was used as a gateway to relay messages from end-device sensors to the network server for data processing and storage. The LoRa network was configured to use a CB of $125 \mathrm{kHz}$ and a fixed SF of 7. The test results showed that the gradual increase in obstacles and distance has a major impact on the reliability of LoRa network. The study, therefore, recommended that while LoRa technology could be used to provide indoor IoT applications, it promises more efficiency when used as indoor solution.

Performance assessment of the LPWAN in smart grid systems based on LoRa technology was presented [21]. The assessment was carried out using a LoRaSim system network simulator which was configured with varying LoRa PHY parameters, such as Transmission Power (TP), Carrier Frequency $(\mathrm{CF}), \mathrm{SF}$ and $\mathrm{CB}$. The simulator used Data Extraction Rate (DER) and Network Energy Consumption (NEC) as the performance indicators for the LoRa deployment. Using the performance results, the authors postulated that the implementation of LoRa technology in distributed smart grids is not a feasible option since a large number of LoRa gateways are needed to manage tremendous traffic from all end nodes. The main concern of the authors was whether the LoRa network can be robust enough to handle all monitoring information from Smart Distributed Grids (SDG) applications including video surveillance which needs high data throughput. However, the authors concluded that the performance results can be considered fairly satisfactory for managing small traffic smart grid applications such as monitoring consumer power consumption data.

The results and key findings from the above related works were used as a benchmark in this study to assess the efficiency of the proposed electricity usage monitoring system for decentralized mini-grids. Compared to previous studies, this study has put much emphasis on low-cost system design and stakeholder-driven system requirements by using available infrastructure and hardware to develop the system.

\section{Proposed System}

In this section, the main features of the proposed integrated LoRa-GPRS electricity usage monitoring system is described in detail. Finally, the methodology used to assess the performance of the system is explained.

\section{A. Proposed System Architecture}

The conceptual framework architecture of the proposed system is shown in Fig. 1. The system incorporates the application of IoT to monitor power consumption information of each smart meter and transmits data to the cloud server.

Consumer power consumption data is collected through a private LoRa network installed between smart meters and a local monitoring centre, which is then transmitted to the cloud server via a GPRS gateway. All decision making logic such as remote connection or disconnection of smart meters, bill processing and user authentication are handled on the cloud server.

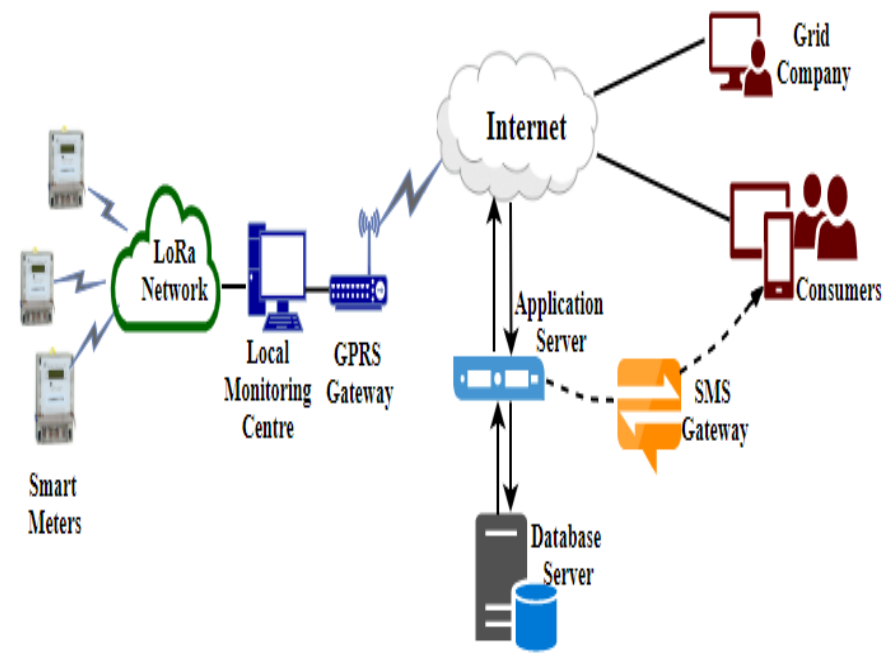

Fig. 1. Proposed System Architecture.

In addition, the Short Message Service (SMS) gateway was integrated into the system to provide consumers with usage notification feature when prepaid electricity units are about to get exhausted.

\section{B. Smart Meter unit Configuration}

The embedded part of the proposed system consists of the smart meter unit. The smart meter unit was designed to have three main features: the ability to accurately measure and record power consumption, the ability to be managed remotely by the utility company and a reliable communication link to the local monitoring centre. Fig. 2 shows the block diagram of the proposed smart meter unit indicating the main components.

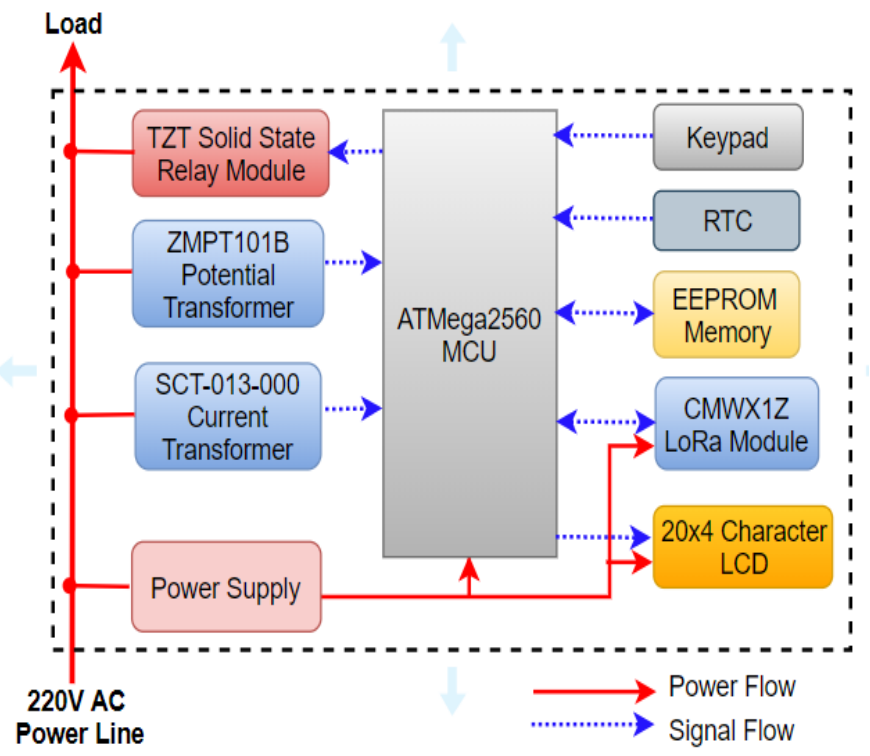

Fig. 2. Block Diagram of the Proposed Smart Meter unit. 
Accurate power consumption measurement is achieved by using efficient but less expensive sensor modules available in the local market. The current sensor is made up of a noninvasive split-core SCT-013-000 current transformer which can withstand currents of up to 100 Amperes. This module has been selected because of its broad current range measurement and flexibility in cabling, as it does not require direct contact with the power cable [22]. Voltage sensor consists of a ZMPT101B single-phase voltage transformer which can withstand up to $250 \mathrm{AC}$ voltage. This module was selected due to its high accuracy, and comes with a built-in trim potentiometer for adjusting the Analogue to Digital Converter (ADC) output.

The smart meter unit is remotely controlled using a TZT solid state relay module that connects or disconnects the consumer load from the main power supply. Additionally, in embedded systems and IoT applications, the Real Time Clock (RTC) is an integrated circuit (IC) which is used to keep the precise time and date of sensor measurements and microprocessor operations to synchronize with the absolute reference time [23]. RTC can be part of the microprocessor IC or an external module connected to the microprocessor via a serial interface. In this study, an external DS1307 RTC module was selected because it has a built-in backup battery that allows time tracking even when there is no primary power supply [24]. For the part of microcontroller unit, after thorough analysis, the Arduino-based ATmega2560 board was selected because of its low cost, ample processing capacity, it has a large number of integrated ADC inputs and is easily available on the local market.

\section{LoRa-GPRS Network}

Each smart meter unit was incorporated with a CMWX1ZZABZ LoRa module (shown in Fig. 3) which operates at the license-free band of $868 \mathrm{MHz}$ and CB of 125 $\mathrm{kHz}$ [25]. The transmission power at this frequency band was limited to $14 \mathrm{dBm}$ in accordance with the LoRa PHY specifications, which makes the signal power prone to attenuation from building blockages and other structures. In order to increase a robustness of the transmitted signal against interference, a high SF factor of 12 was used.

Additionally, LoRa gateway base stations have been installed at the top of a few poles as shown in Fig. 4 in order to boost the signal strength around the mini-grid centre. The modules used in this study belong to the class C LoRa devices, which have the ability to continuously send data to the gateway and listen to downlink messages all the time because they are powered by mains supply from the grid centre, therefore power consumption is not a big issue [26]. Fig. 5 shows a local monitoring center connected to a GPRS gateway to transmit power consumption data to the cloud server via the existing cellular communication network.

Fig. 6 shows the flowchart diagram illustrating the algorithm of the data acquisition unit between LoRa and GPRS modules on how power consumption data from each smart meter unit is collected and sent to the cloud server.

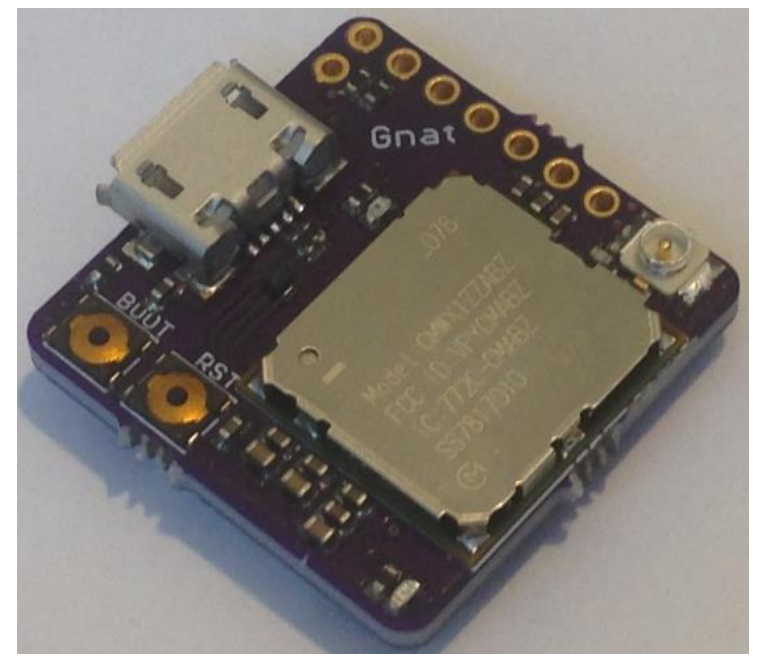

Fig. 3. CMWX1ZZABZ LoRa Module used for Data Transmission between Smart Meter units and Local Monitoring Centre.

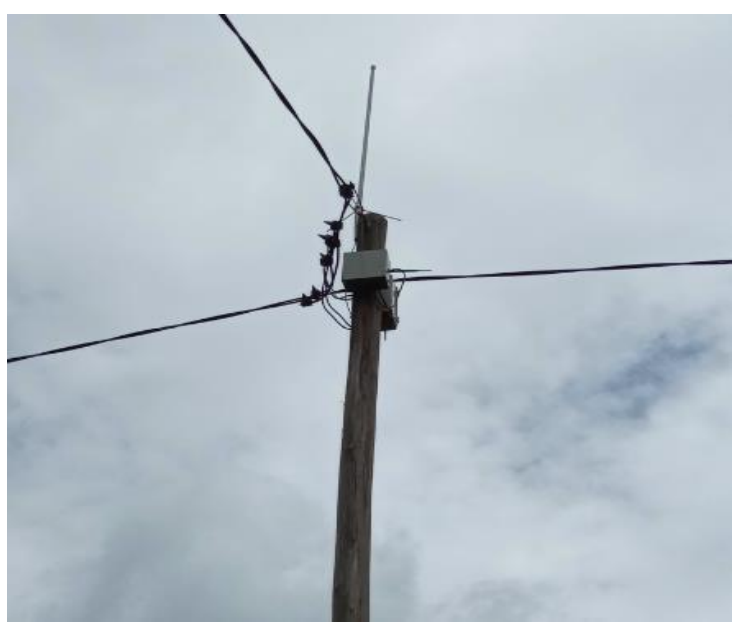

Fig. 4. LoRa Base Station Installed at the Pole.

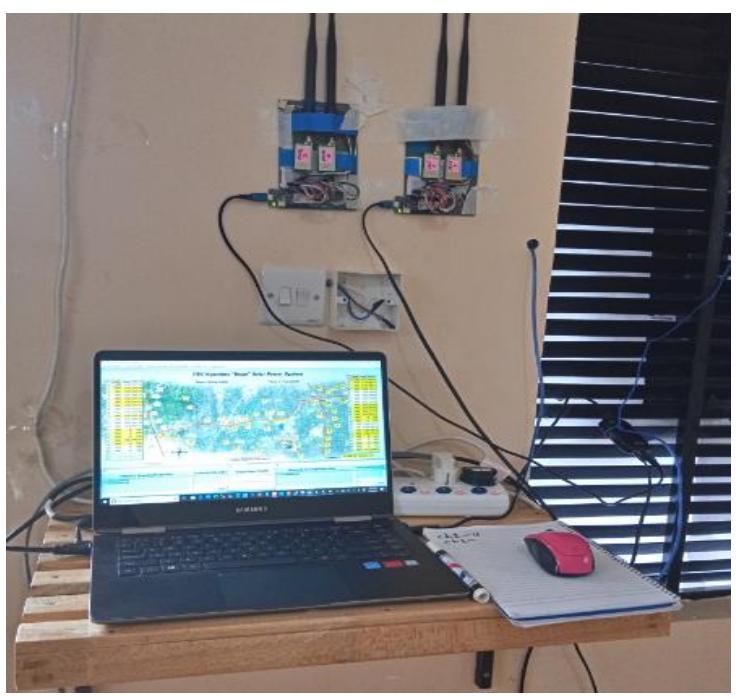

Fig. 5. Local Monitoring Centre and GPRS Gateway. 


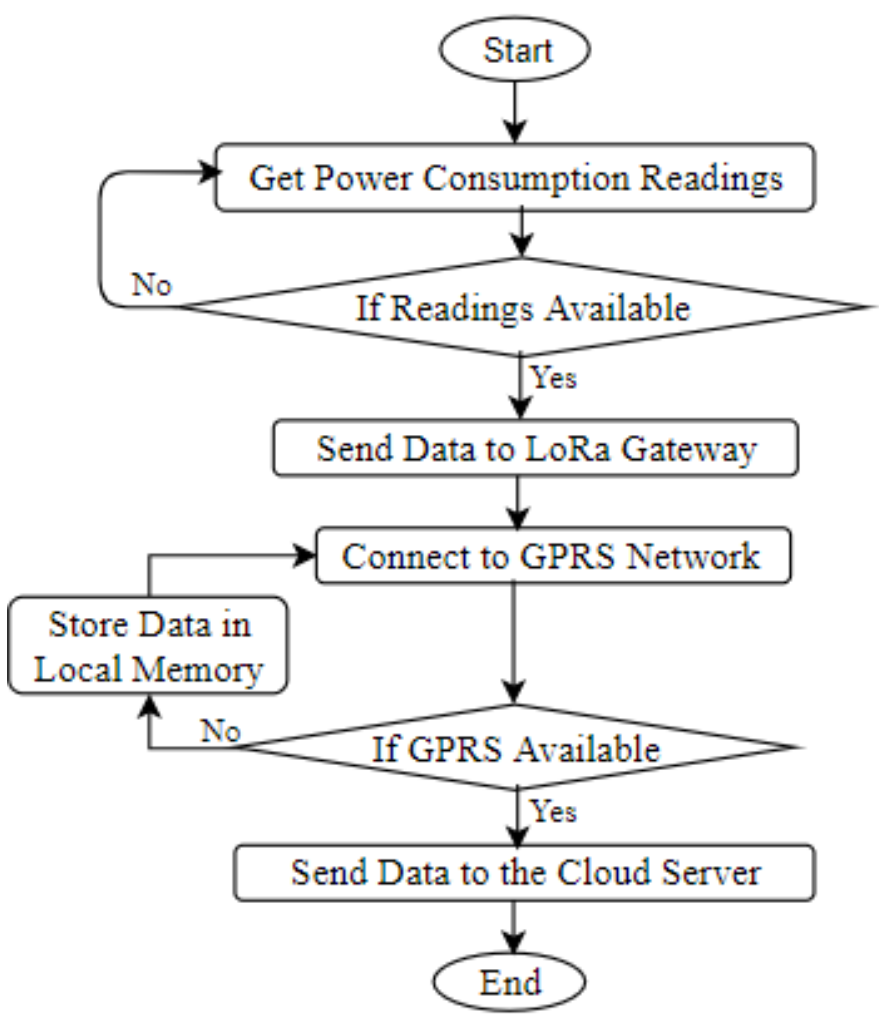

Fig. 6. Flowchart Algorithm of the Data Transmission unit.

\section{Cloud Server Configuration}

In order to minimize the burden of data processing overload between smart meters, the local monitoring center and the cloud server, all the basic computational tasks related to power consumption are carried out locally at the smart meter unit. The cloud server is only responsible for handling data storage, data presentation, usage monitoring and usage notification.

The cloud server was developed by using PHP Laravel and JavaScript frameworks while the database was developed by using MySQL database management system. Fig. 7 illustrates the consumer profile on the cloud server which displays basic information such as consumer contact information, status of the remaining electricity credits and the power supply connection status between smart meter unit and the mini-grid center. Links to other cloud server modules such as customer support, payment system, historical power usage and account settings are located at the bottom of the consumer profile interface.

Fig. 8 shows the historical power consumption information for the previous seven days in a graphical format for the same consumer account. The interface also shows the associated cost of the selected time frame. The cost is expressed in Tanzanian Shillings (TZS.), which is the official local currency of Tanzania where the study was conducted.

\section{Nishati Monitor}

Consumer No: $\mathbf{0 0 0 1 0}$

Account Type: Prepaid

\section{User information}

Consumer Name: Erick Samson

Consumer Email: $\quad$ ericksamjf@gmail.com

Consumer Phone: 0715565852

Remaining Units: $\quad 12.5$

Connetion Status: ON

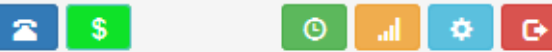

Fig. 7. Cloud Server Consumer Profile user Interface.

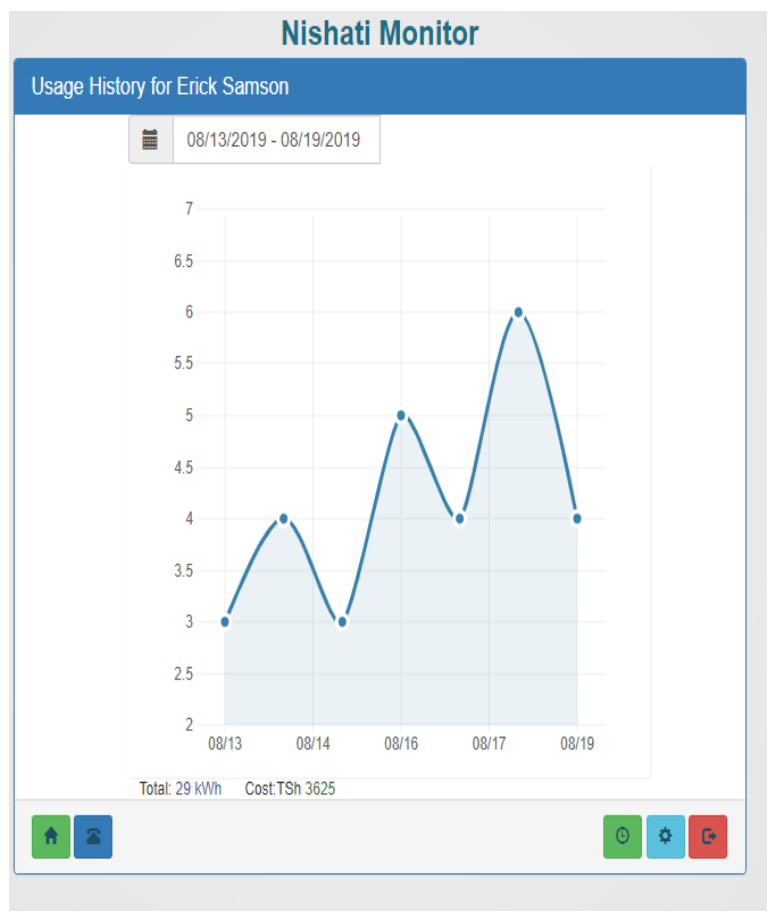

Fig. 8. Cloud Server Historical Power Consumption. 


\section{Performance Evaluation of the Proposed SYSTEM}

In this section, the methodology used to evaluate the performance of the integrated LoRa-GPRS electricity usage monitoring system is described. The aim of this evaluation is to assess the accuracy of the communication infrastructure and whether the proposed system can record and transmit power consumption data with minimum packet loss. To achieve this, a smart meter prototype shown in Fig. 9 was used to measure and record power consumption of various electric appliances for a period of 20 days. Consumption data was then sent to the cloud server via a GPRS gateway for storage and historical access.

In order to validate the accuracy of the data collected on the cloud server, a DDSD101-KT1 digital meter shown in Fig. 10 was used as a reference for comparing the results [27]. This digital meter is a simple and portable power consumption meter that records power consumption in kilowatt-hour units within a $3 \%$ accuracy.

The performance evaluation was conduced using five common home appliances: light bulbs, laptop computer, electric kettle, a small fridge and a radio. These appliances were selected so that the system performance can be assessed on both types of load, linear and non-linear. Incandescent light bulbs, electric kettle and the radio are linear while a laptop computer and fridge are non-linear loads. The power ratings information of these appliances is shown in Table I. Each appliance was connected to the load side of the smart meter prototype and left to run over a certain period of time. The data collected on the cloud server was recorded and the results were compared to the same data set collected using the DDSD101KT reference digital meter over the same time period with the same appliances.

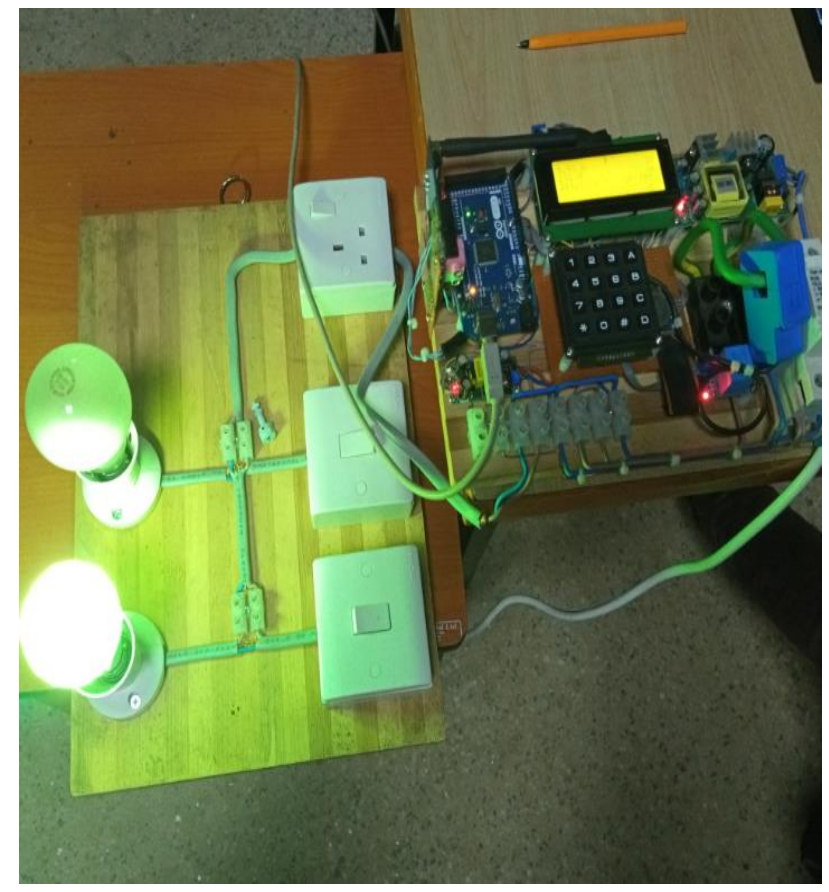

Fig. 9. Power Consumption Measurement on a Smart Meter Prototype.

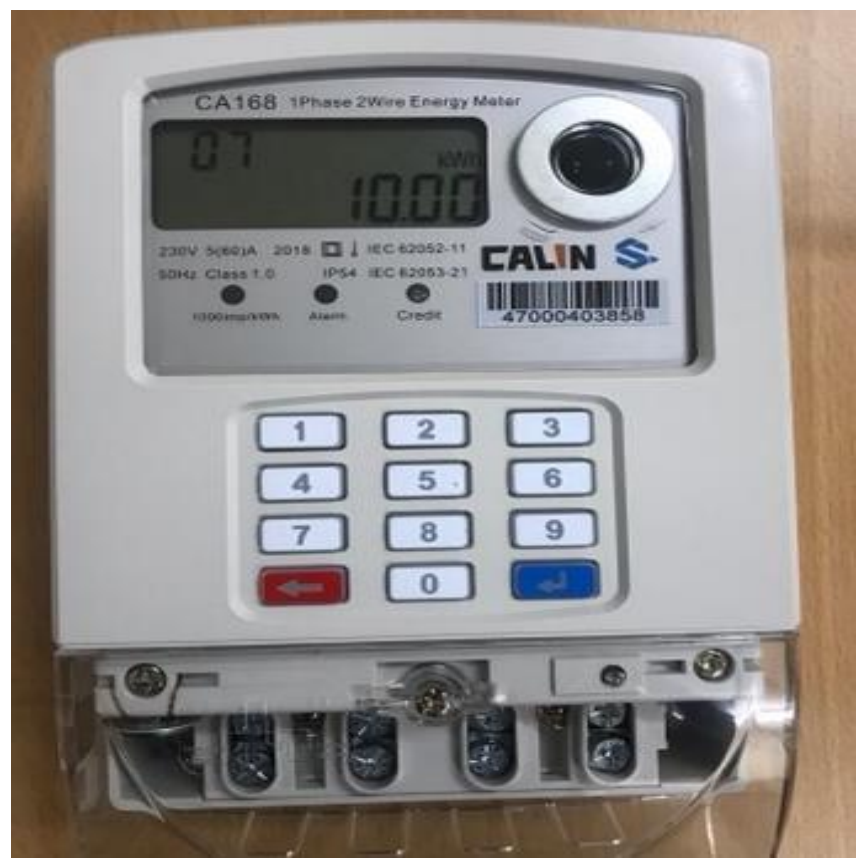

Fig. 10. DDSD101-KT1 Digital Meter used as a Reference.

TABLE. I. POWER RATINGS OF THE SELECTED ElECTRIC APPLIANCES USED FOR THE PERFORMANCE TESTING

\begin{tabular}{|l|l|}
\hline Appliance & Power Rating (Watts) \\
\hline Incandescent Light Bulbs & 220 \\
\hline Laptop Computer & 60 \\
\hline Electric Kettle & 1500 \\
\hline Fridge & 620 \\
\hline Radio & 160 \\
\hline
\end{tabular}

\section{RESUlts AND Discussion}

Compared to other studies [16-21] which used various LoRa network parameters such as node-to-node data processing time, LoS and communication range as the key performance metrics, in this paper a simple approach was used to evaluate the performance of the proposed system. By assessing the accuracy of the power consumption data collected on the cloud server over a specific period of time as the performance metric, it ensures that all other communication variables are taken into account in the final data. In addition, certain performance metrics such as LoS and transmission range may not be extensively varied since smart meters are usually fixed at consumer houses, making parameters adjustment less flexible compared to other IoT applications such as in weather monitoring.

The results of power consumption data from both the cloud server and the digital reference meter are shown in Table II. From the table, the overall measurement performance of the integrated LoRa-GPRS electricity usage monitoring system is generally satisfactory. The proposed system produced a less than $5 \%$ absolute percentage error for all types of electrical appliances used during performance testing, which is within the acceptable standards. 
TABLE. II. COMPARISION OF POWER CONSUMPTION RESULTS BETWEEN THE PROPOSED SySTEM VERSUS THE DDSD101-KT DigitAL METER

\begin{tabular}{|l|l|l|l|l|}
\hline Appliance & $\begin{array}{l}\text { Running } \\
\text { Time } \\
\text { (Hours) }\end{array}$ & $\begin{array}{l}\text { Cloud } \\
\text { Server } \\
\text { Units (kWh) }\end{array}$ & $\begin{array}{l}\text { DDSD101- } \\
\text { KT1 Units } \\
\text { (kWh) }\end{array}$ & $\begin{array}{l}\text { Percentage } \\
\text { Error }(\%)\end{array}$ \\
\hline $\begin{array}{l}\text { Incandescent } \\
\text { Light Bulbs }\end{array}$ & 192.15 & 39.16 & 40.72 & 3.83 \\
\hline Laptop Computer & 102.50 & 5.79 & 6.08 & 4.79 \\
\hline Electric Kettle & 7.92 & 10.91 & 11.26 & 3.15 \\
\hline Fridge & 22.43 & 13.03 & 13.52 & 3.66 \\
\hline Radio & 17.0 & 2.35 & 2.43 & 3.42 \\
\hline
\end{tabular}

The power consumption data recorded on the cloud server were slightly less than the same data recorded on the reference digital meter for all electrical appliances using during the test. This was attributed to the slight packet transmission delays between smart meter unit and the LoRa gateway which was largely caused by large high SF configuration in LoRa PHY layer [12]. High SF values increase the time on the air of the transmitted frames, although the signal strength against interference and communication range is increased.

To mitigate this type of systematic error, the LoRa modules must be configured with SF as low as 7, but this will have a negative impact on the signal transmission range between smart meter units and gateway base stations. A trade-off therefore needs to be made between signal quality and transmission range, but at the expense of the data throughput.

\section{CONCLUSION}

In this paper, a performance evaluation of the cost-effective LoRa-GPRS integrated electricity usage monitoring for decentralized mini-grid systems was presented. Because of their decentralized nature, most mini-grid centers are located in remote areas with limited ICT infrastructure, hence it is typically not the best option to rely solely on public networks to monitor the smart meters. The combination of LPWAN and conventional cellular networks provides a better solution for the management of decentralized mini-grid centers. In this study, the performance evaluation of the proposed system was conducted by comparing the results obtained from the cloud server with the reference data collected locally using a standard digital meter.

This study found that the proposed system had satisfactory performance results promising its potential application for IoT monitoring in decentralized grids. Although the collected power consumption data were always slightly lower compared to the expected values, the percentage error was within the acceptable standards. To increase the accuracy of the data collected, LoRa modules need to be configured with lower SF values, which will also require additional cost of installing more LoRa gateways throughout the mini-grid centre. As a future work, the integrated mini-grid communication infrastructure needs to be improved in order to enable monitoring of more parameters such as power quality, power theft, Time of Use (ToU) pricing and event reporting.

\section{ACKNOWLEDGMENT}

This work was supported by the African Development Bank (AfDB), United Republic of Tanzania, through project No. P-Z1-IA0-016 and grant No. 2100155032816.

\section{REFERENCES}

[1] Hartvigsson E, Ehnberg J, Ahlgren E, Molander S. Assessment of load profiles in minigrids: A case in Tanzania. Proc Univ Power Eng Conf. 2015;15:1-5.

[2] Chaplin D, Mamun A, Protik A, Schurrer J, Vohra D, Bos K, et al. Grid Electricity Expansion in Tanzania: Findings from a Rigorous Impact Evaluation. Mathematica Policy Research. 2017.

[3] Ahlborg H, Hammar L. Drivers and barriers to rural electrification in Tanzania and Mozambique - grid extension, off-grid and renewable energy sources. 2011.

[4] Khadar Associate Professor AA, Ahamed Khan Professor J, MIT Madanpalli E, S Nagaraj Professor IM. Research Advancements Towards in Existing Smart Metering over Smart Grid [Internet]. Vol. 8, IJACSA) International Journal of Advanced Computer Science and Applications. 2017 [cited 2018 Oct 30]. Available from: www.ijacsa.thesai.org.

[5] Chattopadhyay D, Bazilian M, Lilienthal P. More power, less cost: Transitioning up the solar energy ladder from home systems to minigrids. Electr J [Internet]. 2015;28(3):41-50. Available from: http://dx.doi.org/10.1016/j.tej.2015.03.009.

[6] Persia S, Carciofi C, Faccioli M. NB-IoT and LoRA connectivity analysis for M2M/IoT smart grids applications. 2017 AEIT Int Annu Conf Infrastructures Energy ICT Oppor Foster Innov AEIT. 2017.

[7] Nielsen JJ, Madueño GC, Pratas NK, Sørensen RB, Stefanovi' C, Popovski P. What Can Wireless Cellular Technologies do about the Upcoming Smart Metering Traffic? IEEE Commun Mag - Commun Stand Suppl. 2015;(June):41-7.

[8] Cheng Y, Saputra H, Goh LM, Wu Y, Tower CS, Way F. Secure Smart Metering Based on LoRa Technology. 2018.

[9] Al-Fuqaha A, Guizani M, Mohammadi M, Aledhari M, Ayyash M. Internet of Things: A Survey on Enabling Technologies, Protocols, and Applications. IEEE Commun Surv Tutorials. 2015;17(4):2347-76.

[10] Yuan Z, Jin J, Sun L, Chin KW, Muntean GM. Ultra-Reliable IoT Communications with UAVs: A Swarm Use Case. IEEE Commun Mag. 2018;56(12):90-6.

[11] Sinha RS, Wei Y, Hwang SH. A survey on LPWA technology: LoRa and NB-IoT. ICT Express [Internet]. 2017;3(1):14-21. Available from: http://dx.doi.org/10.1016/j.icte.2017.03.004.

[12] Adelantado F, Vilajosana X, Tuset-Peiro P, Martinez B, Melia-Segui J, Watteyne T. Understanding the Limits of LoRaWAN. IEEE Commun Mag. 2017;55(9):34-40.

[13] Mekki K, Bajic E, Chaxel F, Meyer F. A comparative study of LPWAN technologies for large-scale IoT deployment. ICT Express [Internet]. 2019;5(1):1-7. Available from: https://doi.org/10.1016/j.icte.2017.12.005

[14] Li S, Raza U, Khan A. How Agile is the Adaptive Data Rate Mechanism of LoRaWAN? 2018 IEEE Glob Commun Conf GLOBECOM 2018 Proc. 2018;

[15] Cattani M, Boano CA, Römer K. An experimental evaluation of the reliability of lora long-range low-power wireless communication. J Sens Actuator Networks. 2017;6(2).

[16] Petäjäjärvi J, Mikhaylov K, Pettissalo M, Janhunen J, Iinatti J. Performance of a low-power wide-area network based on lora technology: Doppler robustness, scalability, and coverage. Int J Distrib Sens Networks. 2017;13(3).

[17] Campo G, Gomez I, Calatrava S, Martinez R, Santamaria A. Power Distribution Monitoring Using LoRa: Coverage Analysis in Suburban Areas. 2018;233-8.

[18] Augustin A, Yi J, Clausen T, Townsley WM. A Study of LoRa : Long Range \& Low Power Networks for the Internet of Things. 2016;1-18. 
[19] Zanella A, Zorzi M. L ong -R ange C ommunications in U nlicensed B ands: $\mathrm{T}$ he $\mathrm{R}$ ising $\mathrm{S}$ tars in the $\mathrm{I}$ o $\mathrm{T}$ and $\mathrm{S}$ mart $\mathrm{C}$ ity $\mathrm{S}$ cenarios. 2016;(October):60-7.

[20] Ghiasimonfared A, Righini D, Marcuzzi F, Tonello AM. Development of a Hybrid LoRa / G3-PLC IoT Sensing Network : an Application Oriented Approach. 2017;(October):503-8.

[21] Haidine A, Aqqal A, Dahbi A. Performance Evaluation of Low-Power Wide Area based on LoRa Technology for Smart Metering. 2018 6th Int Conf Wirel Networks Mob Commun. 2018;1-6.

[22] Abubakar I, Khalid SN, Mustafa MW, Shareef H, Mustapha M. Calibration of ZMPT101B voltage sensor module using polynomial regression for accurate load monitoring. ARPN J Eng Appl Sci. 2017;12(4):1076-84.
[23] Barai GR, Krishnan S, Venkatesh B. Smart metering and functionalities of smart meters in smart grid - A review. 2015 IEEE Electr Power Energy Conf Smarter Resilient Power Syst EPEC 2015. 2016;138-45.

[24] ElectronicWings. Sensors Modules Real Time Clock Rtc Ds1307 Module | Sensors Modul... [Internet]. 2018 [cited 2019 Nov 10]. Available from: https://www.electronicwings.com/sensors-modules/real-time-clock-rtcds1307-module.

[25] Hackaday. Project | Hackable CMWX1ZZABZ (LoRa) Devices | Hackaday.io [Internet]. 2018 [cited 2019 Nov 11]. Available from: https://hackaday.io/project/35169/logs.

[26] Eric B. LoRa - LoRa documentation [Internet]. 2018. 2018 [cited 2019 Dec 19]. Available from: https://lora.readthedocs.io/en/latest/\#.

[27] Wasion. DDSD101-KT1 Single Phase Electric Energy Meter [Internet]. 2019 [cited 2019 Dec 21]. Available from: http://www.wasion.com/ $\mathrm{EN} / \mathrm{pro} / 484 . \mathrm{html}$. 